\title{
Iranian general populations' and health care providers' preferences for benefits and harms of statin therapy for primary prevention of cardiovascular disease
}

\author{
Hassan Saadati ${ }^{1}$, Hamid Reza Baradaran ${ }^{1,2,3^{*}}$, Goodarz Danaei ${ }^{4}$, Afshin Ostovar ${ }^{5}$, Farzad Hadaegh ${ }^{6}$, Leila Janani ${ }^{7}$, \\ Ewout W. Steyerberg ${ }^{8,9}$ and Davood Khalili ${ }^{6,10^{*}}$ (1)
}

\begin{abstract}
Background: The use of statins for primary prevention of cardiovascular diseases is associated with different benefit and harm outcomes. The aime of this study is how important these outcomes are for people and what people's preferences are.

Methods: We conducted a preference-eliciting survey incorporating a best-worst scaling (BWS) instrument in Iran from June to November 2019. The relative importance of 13 statins-related outcomes was assessed on a sample of 1085 participants, including 913 general population (486 women) and 172 healthcare providers from the population covered by urban and rural primary health care centers. The participants made trade-off decisions and selected the most and least worrisome outcomes concurrently from 13 choice sets; each contains four outcomes generated using the balanced incomplete block design.
\end{abstract}

Results: According to the mean (SD) BWS scores, which can be $(+4)$ in maximum and $(-4)$ in minimum, in the general population, the most worrisome outcomes were severe stroke (3.37 (0.8)), severe myocardial infarction (2.71(0.7)), and cancer $(2.69(1.33))$. While myopathy $(-3.03(1.03))$, nausea/headache $(-2.69(0.94))$, and treatment discontinuation due to side effects $(-2.24(1.14))$ were the least worrisome outcomes. Preferences were similar between rural and urban areas and among health care providers and the general population with overlapping uncertainty intervals.

Conclusion: The rank of health outcomes may be similar in various socio-cultural contexts. The preferences for benefits and harms of statin therapy are essential to assess benefit-harm balance when recommending statins for primary prevention of cardiovascular diseases.

Keywords: Preferences, Statins, Benefit harm outcomes, Primary prevention, Cardiovascular disease

*Correspondence: baradaran.hr@iums.ac.ir; hamid.baradaran@abdn.ac.uk. dkhalili@endocrine.ac.ir; dkhalili@hsph.harvard.edu

${ }^{1}$ Department of Epidemiology, School of Public Health, Iran University

of Medical Sciences, Tehran, Iran

${ }^{6}$ Prevention of Metabolic Disorders Research Center, Research Institute for Endocrine Sciences, Shahid Beheshti University of Medical Sciences, Tehran, Iran

Full list of author information is available at the end of the article

\section{Background}

Cardiovascular diseases contribute to over $30 \%$ of deaths worldwide and more than $40 \%$ in Iran $[1,2]$. Given that the raised total cholesterol and low-density lipoprotein (LDL) cholesterol are the major risk factors for cardiovascular disease (CVD), controlling hypercholesterolemia is an important target in managing CVD risk [3]. Statins are a class of lipid-lowering drugs that have been widely 
used for reducing the level of plasma LDL cholesterol [4]. Statins are widely prescribed for the primary and secondary prevention of CVD $[5,6]$, and their use has been significantly increased over the last 30 years [7].

The various harm and benefit outcomes that are associated with using statins have been reported in previous studies [8-10]. Shared decision-making is a process in which patients and physicians work together to make the best decision for health care, especially when treatments have different benefit and harm outcomes [11]. Statin therapy is a crux for primary prevention of CVD, and its prescription is a preference-sensitive decision [12]. Guidelines provide tools for risk calculation and decision thresholds for preventive drug therapy and recommend shared decision for borderline CVD risks [13, 14]. People value the benefit and harm outcomes of statins differently. This study aimed to elicit the preferences for these benefits and harms in different settings, including rural and urban areas and among healthcare providers and the general population. We used the best-worst scaling technique to elicit preferences; it is a popular method in health for its cognitive and administrative simplicity [15].

\section{Methods}

We conducted a preference-eliciting survey incorporating a best-worst scaling (BWS) instrument in Iran from June to November 2019. The best-worst scaling method was devised by Finn and Louviere [16] and introduced to healthcare research by McIntosh and Louviere [17]. In this method, choice sets are constructed by combining various items and then asking respondents to select the best and worst items from each choice set. According to the choice sets format, BWS is divided into three types: the object case, the profile case, and the multi-profile case $[18,19]$. Because in the present study, our purpose was to measure the individual preferences, we used object case, which is suitable for perceiving the relative evaluation of the multiple items the respondents chose.

\section{Questionnaire design and procedure}

In the object case BWS - or maximum difference scaling (MaxDiff) [16], measuring a set of items on an underlying, latent, subjective scale is interested. Thus, this case requires a list of items to be measured. In the current study, we used a list of items consist of 13 statinassociated benefit/harm outcomes including moderate MI, severe MI, moderate stroke, severe stroke, unstable angina, heart failure, liver injury, myopathy, type 2 diabetes, acute kidney failure, cancer, nausea/headache, and treatment discontinuation due to side-effects. These outcomes have been previously selected from RCTs/ meta-analysis by Yebyo et al. [8] To equally familiarize respondents with each of the 13 outcomes, we prepared specific clear definitions and short lay descriptions for each outcome. To assess whether the short lay descriptions were clear enough for the individuals, we first consulted experts in the field to check for their appropriateness. Second, we piloted the lay descriptions with 20 individuals aged 40-60 years that helped rephrase and simplify the medical terms; only some minor changes were made in the wording and no substantial revisions were required.

The object case BWS method consists of a series of choice sets, from the list of items, where respondents are asked to select the "best" (or most important) and "worst" (or least important) items in each choice set. In the present study, the terms "best" and "worst" refer to the most and least worrisome clinical outcome, respectively, and respondents are asked to indicate the most and the least worrisome outcome in each choice set. A balanced incomplete block design was used to construct the choice sets so that each possible choice was seen the same number of times through all choice sets, and each choice set included the same number of items [19]. Therefore, each choice has the same possibility to be chosen as the most or least worrisome outcome. This design generated 13 choice sets with four outcomes in each choice set so that each outcome coexisted with another one just once (Appendix). An example of BWS questions is given in the appendix. A preliminary test with 10 participants was conducted to assay the feasibility of the survey design.

\section{Participants and data collection}

Participants were selected from the population covered by primary health care centers in Iran from June to November 2019. We decided IraPEN piloted cities located in four provinces: Naghadeh in West Azerbaijan, Maragheh in East Azerbaijan, Shahreza in Isfahan, and Baft in Kerman Province. IraPEN is an Iranian modified version of the WHO Package of Essential Noncommunicable Diseases Interventions (WHO PEN). It is a part of the national health transformation plan, launched in 2014 by the Ministry of Health to provide universal health coverage, including access to CVD prevention and care [20]. In each city, four urban and four rural health centers were randomly selected. In Maragheh, the number of centers chosen was twice that of the other three cities because the population covered by primary health care centers was almost twice that of the other areas. We planned to recruit 1000 (500 in rural health centers) carereceivers attending to these health centers.

We recruited the participants using the household health files from health centers with a random sampling plan. Participants recruited were 40 years or older without a history of CVD events. The study participants were interviewed face to face in health centers. First, 
to familiarize participants with the 13 outcomes, simple descriptions were presented and asked respondents to express their perceived severity using a visual analog scale (VAS) for each of the 13 outcomes. We then provided the choice sets designed using the BWS method. In each BWS choice set, the respondent was asked to select the most and least worrisome outcome. We asked all staff at selected health centers to answer the same questionnaire as well. A total of 172 health care providers from urban and rural health centers completed the BWS questionnaire. All participants signed an informed consent form and ethical approval for this study was obtained from the Ethics Committee of the Iran University of Medical Sciences.

\section{Statistical analysis}

We followed the standard analysis of the BWS designs and used a counting approach, followed by statistical modeling, to analyze data [21]. The counting approach calculates several types of scores based on the number of times each item was chosen as "best" and the number of times chosen as "worst" across participants. To analyze item importance, we used the average of "Best minus Worst (B-W)".

The modeling approach, known as the Maximum-difference model (logit model), is an expansion of the conditional logit model. This model assumes that respondents evaluate all possible pairs of items and choose the pair that reflects the maximum difference in preference or importance. Under these assumptions, the probability of selecting the item " $\mathrm{i}$ " as the "best" and item " $\mathrm{j}$ " as the "worst" expressed as a conditional logit model. In our study, each choice set contained four of 13 outcomes. Therefore, the number of possible pairs in each choice set was 12 pairs. The demographic and clinical characteristics of respondents were reported using descriptive statistics. Also, linear regression was used to evaluate factors, among these characteristics, that affected the preferences. We used R 3.2.2 and STATA 14.0 for data analyses.

\section{Results}

\section{Characteristics of respondents}

We invited 1000 care-receivers from 20 urban and 20 rural health centers. The non-response rate was less than $5 \%$, those with a history of CVD were excluded, and finally, 913 subjects (449 from urban and 464 from rural) were eligible and included in the study. All 172 invited caregivers participated in the study. The sociodemographic and other characteristics of participants are summarized in Table 1. The mean (SD) age of the total participants was $49.8(8.1)$ years and $53.2 \%(\mathrm{n}=486)$ were female. In terms of education, $97 \%$ of the general population participants were literate and $50 \%$ educated in middle school or higher (60\% in urban and 35\% in rural areas). Among the general population, $19.6 \%$ reported that they used statins at the time of study or in the past. The mean (SD) age of 172 health care providers was 35.4 (7.5) years, and $82.5 \%(n=142)$ were female. The health care providers' demographic and clinical characteristics were different from those of the general population.

\section{Best- worst scaling survey results}

The counting analysis results for each group (health care provider, total population, and subpopulations) are presented in Table 2 as total best, total worst, and best minus worst (B-W) score for each outcome. According to the $\mathrm{B}-\mathrm{W}$ scores, the most worrisome outcomes were severe stroke, sever MI, and cancer, and the least worrisome outcomes were myopathy, nausea/headache, and treatment discontinuation.

Figure 1 shows more information regarding the relative importance of outcomes among individuals. Based on the means of the B-W scores of outcomes as well as their standard deviations, severe MI and cancer are similarly important, but the variance of cancer is more remarkable than severe MI; therefore, the importance of cancer differs largely among individuals. Moreover, severe stroke has a relatively high mean with a relatively low standard deviation of the score, which means that the majority mark severe stroke as the most problematic outcome. Bar plots were drawn from $\mathrm{B}-\mathrm{W}$ scores to show the heterogeneity in more detail (Additional file 1: Figure 2).

Estimates of statin outcome importance, using the VAS score (bounded by 0 and 1), are presented in Additional file 1: Table 3. Box plots of the BWS scores (on a scale from -4 to 4 ) and the VAS scores (on a scale from $0-1$ ) are shown in Fig. 2. The ranking of the 13 outcomes by the median of individual BWS was similar to that of VAS. However, as the figure depicts, there is less overlap in the BWS distributions than the VAS ones and the inconsistency of the scores based on the VAS is broader than that based on the BWS.

Table 3 shows the relative importance of the 13 outcomes associated with statin use estimated by the Maximum-difference model. The importance of each outcome was assessed relative to the "Myopathy," which was consistently rated as the least worrisome outcome. The results revealed that severe stroke, sever MI, and cancer, which are the most worrisome outcomes of the 13 outcomes associated with statin use in the general population, are approximately 905,349 , and 318 times as worrisome as myopathy, respectively (these numbers are exponential of the coefficients in Table 3).

Table 4 presents data on self-reported difficulties in understanding and compilation of the BWS questionnaire. Most of the participants rated the BWS task 
Table 1 Characteristics of participants involved in the preference eliciting study

\begin{tabular}{|c|c|c|c|c|}
\hline Characteristics & $\begin{array}{l}\text { Health care provider } \\
n=172 N(\%)\end{array}$ & $\begin{array}{l}\text { Total population } \\
\mathrm{n}=913 \mathrm{~N}(\%)\end{array}$ & $\begin{array}{l}\text { Urban population } \\
n=449 \mathrm{~N}(\%)\end{array}$ & $\begin{array}{l}\text { Rural population } \\
n=464 \mathrm{~N}(\%)\end{array}$ \\
\hline Sex male & $30(17.44)$ & $427(46.77)$ & $201(44.77)$ & $226(48.71)$ \\
\hline Female & $142(82.56)$ & $486(53.23)$ & $248(55.23)$ & $238(51.29)$ \\
\hline Age mean (SD) & $35.37(7.58)$ & $49.78(8.148)$ & $50.26(8.28)$ & $49.31(7.99)$ \\
\hline $40-59$ & $172(100.00)$ & $783(85.76)$ & $381(84.86)$ & $402(86.64)$ \\
\hline$=>60$ & $0(0)$ & $130(14.24)$ & $68(15.14)$ & $62(13.36)$ \\
\hline \multicolumn{5}{|l|}{ Education } \\
\hline Non & $0(0)$ & $29(3.18)$ & $11(2.45)$ & $18(3.88)$ \\
\hline Primary & $10(5.81)$ & $451(49.40)$ & $168(37.42)$ & $283(60.99)$ \\
\hline Middle school & $34(19.77)$ & $272(29.79)$ & $150(33.41)$ & $122(26.29)$ \\
\hline High school and above & $128(74.42)$ & $161(17.63)$ & $120(26.73)$ & $41(8.84)$ \\
\hline \multicolumn{5}{|l|}{ Job } \\
\hline Salaried & $159(92.44)$ & $121(13.25)$ & $87(19.38)$ & $34(7.33)$ \\
\hline Run own business & $0(0)$ & $250(27.38)$ & $99(22.05)$ & $151(32.54)$ \\
\hline Pensioned & $0(0)$ & $107(11.72)$ & $67(14.92)$ & $40(8.62)$ \\
\hline No job & $13(7.56)$ & $435(47.56)$ & $196(43.56)$ & $239(51.51)$ \\
\hline \multicolumn{5}{|l|}{ Co-living person } \\
\hline Alone & $7(4.07)$ & $18(1.97)$ & $8(1.78)$ & $10(2.16)$ \\
\hline Family & 165 (95.93) & $895(98.03)$ & 441 (98.22) & $454(97.85)$ \\
\hline \multicolumn{5}{|l|}{ Morbidity ${ }^{a}$} \\
\hline None & $158(91.86)$ & $631(69.11)$ & $300(66.82)$ & $331(71.34)$ \\
\hline Yes & $14(8.14)$ & $282(30.89)$ & 149 (33.18) & $133(28.66)$ \\
\hline \multicolumn{5}{|l|}{ Statin use } \\
\hline no & $164(95.35)$ & 739 (80.94) & $356(79.29)$ & $383(82.54)$ \\
\hline Yes now & $4(2.33)$ & $123(13.47)$ & $62(13.81)$ & $61(13.15)$ \\
\hline Yes past & $4(2.33)$ & $51(5.59)$ & $31(6.90)$ & $20(4.31)$ \\
\hline Marital status single & $39(22.67)$ & $28(3.07)$ & $8(1.78)$ & $20(4.31)$ \\
\hline Married & $127(73.84)$ & $842(92.22)$ & $425(94.65)$ & 417 (89.87) \\
\hline Divorced & $5(2.91)$ & $12(1.31)$ & $6(1.34)$ & $6(1.29)$ \\
\hline Widow & $1(0.58)$ & $31(3.40)$ & $10(2.23)$ & $21(4.53)$ \\
\hline \multicolumn{5}{|c|}{ Family history of heart disease } \\
\hline Yes & $41(23.84)$ & $254(27.82)$ & $138(30.73)$ & $116(25.00)$ \\
\hline No & $131(76.16)$ & $654(71.63)$ & $309(68.82)$ & $345(74.35)$ \\
\hline Don't know & $0(0)$ & $5(0.55)$ & $2(0.45)$ & $3(0.65)$ \\
\hline \multicolumn{5}{|l|}{ Family history of stroke } \\
\hline Yes & $11(6.40)$ & 125 (13.69) & $60(13.36)$ & $65(14.01)$ \\
\hline No & $161(93.60)$ & $784(85.87)$ & $388(86.41)$ & $396(85.34)$ \\
\hline Don't know & $0(0)$ & $4(0.44)$ & $1(0.22)$ & $3(0.65)$ \\
\hline \multicolumn{5}{|c|}{ Family history of hypertension } \\
\hline Yes & $104(60.47)$ & $518(56.74)$ & $231(51.45)$ & $287(61.85)$ \\
\hline No & $68(39.53)$ & $390(42.72)$ & $215(47.88)$ & $175(37.72)$ \\
\hline Don't know & $0(0)$ & $5(0.55)$ & $3(0.67)$ & $2(0.43)$ \\
\hline \multicolumn{5}{|c|}{ Family history of Hyperlipidemia } \\
\hline Yes & $76(44.19)$ & $291(31.87)$ & $129(28.73)$ & $162(34.91)$ \\
\hline No & $96(55.81)$ & $612(67.03)$ & $316(70.38)$ & $296(63.79)$ \\
\hline Don't know & $0(0)$ & $10(1.10)$ & $4(0.89)$ & $6(1.29)$ \\
\hline \multicolumn{5}{|l|}{ Family history of diabetes } \\
\hline Yes & $50(29.07)$ & $295(32.31)$ & $152(33.85)$ & $143(30.82)$ \\
\hline No & $122(70.93)$ & $616(67.47)$ & $296(65.92)$ & $320(68.97)$ \\
\hline Don't know & $0(0)$ & $2(0.22)$ & $1(0.22)$ & $1(0.22)$ \\
\hline
\end{tabular}

a Hypertension, Hyperlipidemia, Type 2 diabetes, and Cancer 
as easy-to-understand and easy-to-answer. They also reported that answers to all questions were, in a way, consistent with their preferences. Participants stated that the reasons that influence their choices were "Availability of medical care", "Severity of the clinical outcome", "Curability of the clinical outcome", "Long-term consequences of the clinical outcome", and "Cost-effectiveness of medical services" in order. Also, in the multiple linear regression model used to evaluate associations between respondent characteristics and choosing an outcome as the most worrisome, we did not find any significant association between the factors and the outcome preferred (Additional file 1: Table 4).

\section{Discussions}

Hypercholesterolemia is a major and modifiable risk factor for CVD, and medication to lower LDL cholesterol is a crucial recommendation for preventing CVD in many guidelines. We studied the importance of different health outcomes related to statin therapy from the health care providers' and the general population's perspective in urban and rural areas. Each outcome's quantitative weight was derived, showing that the outcomes are scored and ranked similarly in various socio-cultural contexts.

Statin therapy is the first line of treatment for the primary prevention of CVD based on the absolute risk [22, 23]. However, the threshold of absolute risk of CVD over ten years has lowered to $7.5-10 \%$ for this medication $[13,24]$. This decrease in the threshold has increased the number of people eligible for statin therapy that most of them may be healthy and statins may not be appropriate for them. Although using statins in individuals at risk can prevent cardiovascular disease, there are risks of different harm outcomes like muscle pain, increasing serum blood sugar, etc. [8]. Assessing relative importance for treatment outcomes is the key step in doing a benefit-harm assessment, informed by patient preferences [25].

Patients value different health outcomes at different importance. An individual's preference reflects the degree of his/her subjective satisfaction, distress, or desirability for a given health outcome [26]. The tradeoffs between different benefits and harms of treatments are thus largely influenced by how patients place the relative importance on each outcome [27].

We found that people without a history of CVD events considered some statin-related outcomes more worrying than others. Severe stroke, severe MI, and cancers were ranked as the most worrisome outcomes, while treatment discontinuation, nausea/headache, and myopathy were considered the least. There were essentially no differences in the ranking by health care providers and the general population. Also, we found that respondents' preferences in urban and rural areas were similar, despite large differences in the socio-economic contexts. These results were the same as the study by Yebyo et al. carried out in Ethiopia and Switzerland. [8]. They found similar preferences between Ethiopia's and Switzerland's population with overlapping uncertainty intervals. Although more studies in different countries are recommended to ensure these results' generalizability, it seems that the preferences are consistent across dissimilar settings.

Assessing individuals' preferences provides valuable information in promoting preventive campaigns. There has been growing interest in using preference elicitation methods to inform health policy and medical decisionmaking in recent years $[28,29]$. Our results help distinguish between more and less worrying outcomes from the patients' perspective and inform decision-making on the preventive treatment of individuals with risk for cardiovascular events. In this way, quantitative weights can be used to calculate a net benefit for statin therapy. Yebyo et al. used this kind of weights and found the optimum risk thresholds for statin therapy. For instance, they found that at the risk threshold between 12 and $21 \%$, depending on age and sex, weighted harms and benefits of statin therapy are equal for patients based on their own preferences [30].

Although the main economic evaluation method is cost-utility analysis, including the standard gamble and time trade-off, such an approach is not suitable to find trade-offs between health attributes to evaluate current practices. Ordinal preference elicitation methods, including Discrete Choice Experiments and ranking methods, are therefore commonly used in health economics and health service research [31]. The BWS is relatively simple to understand and reduce the cognitive burden for respondents and facilitates the evaluation of maximum-difference questions [15]. BWS also overcomes the traditional 'pick one' task used in Discrete Choice Experiments by eliciting additional information on both the most and least preferred option [32]. Additionally, BWS possesses the ability to embrace a larger set of factors to determine preferences [33]. However BWS has some limitations such as questions about its theoretical foundations, uncertainty about its ability to predict consumer choice, getting repetitive for some if many objects/attributes, and relatively burdensome to some respondents [34].

Compared to the VAS, the BWS is more suitable for ranking the outcomes' relative importance. One advantage of using BWS to elicit preferences is that it allowed us to ask patients in a way that they can make trade-offs between outcomes [16]. In contrast, there are no tradeoffs involved in VAS tasks when doing it and maybe less 


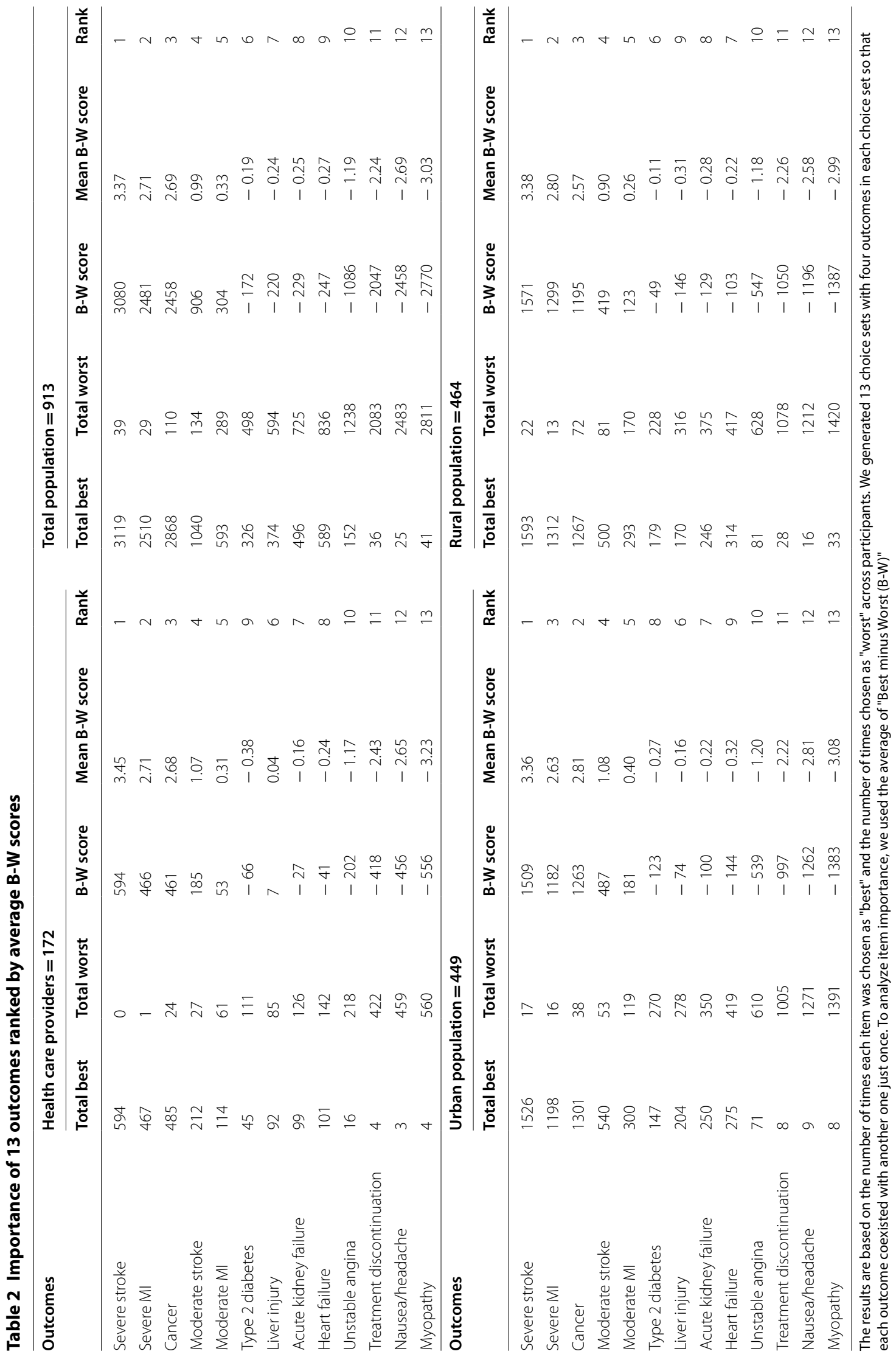



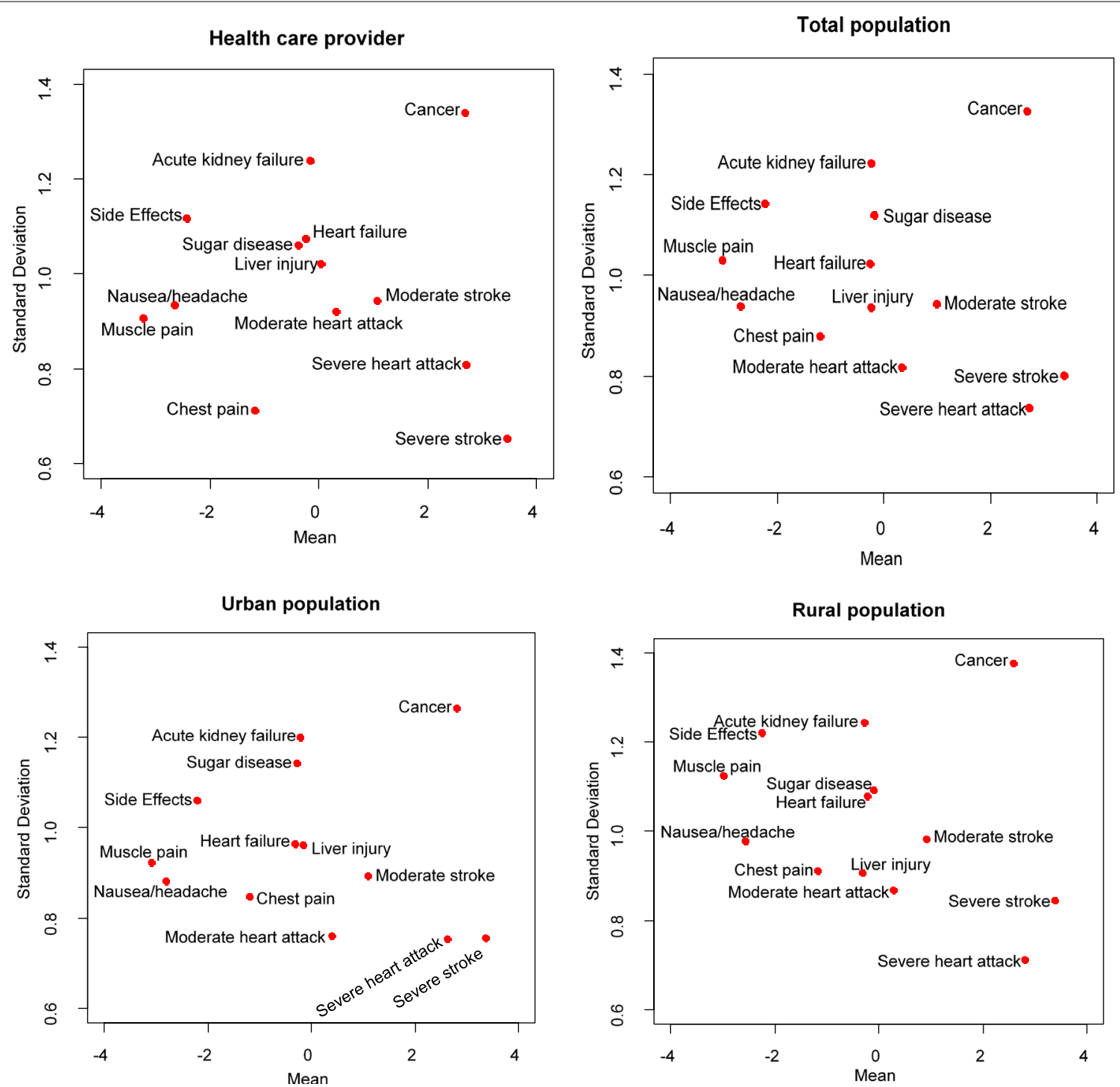

Fig. 1 Relationship between the mean Best-Worst score and its standard deviation. The figure depicts the relative importance of the outcomes among individuals according to the B-W scores' means and standard deviations. The higher the mean and lower the variance, the higher and more stable the importance ranking

sensitive to detect differences in the outcomes' scores [29]. In other words, most patients can complete BWS tasks to express their preferences without major problems. Our results also showed that VAS is lesser reliable with broader distribution in comparison to BWS.

The main limitation in using the BWS method is that a different understanding of the outcomes would influence eliciting the preferences in this approach [28]. Therefore, one of the challenges when designing the questionnaire was to ensure that respondents had a common understanding of the study outcomes when they were doing preference-elicitation tasks. For this purpose, we asked respondents to read the short lay descriptions for each outcome carefully and complete the VAS tasks before they did the BWS tasks. Considering participants from health care centers, and not both public and private sections, is a limitation in our study. However, we showed that individual characteristics, including education and job, had no association with their preferences. 


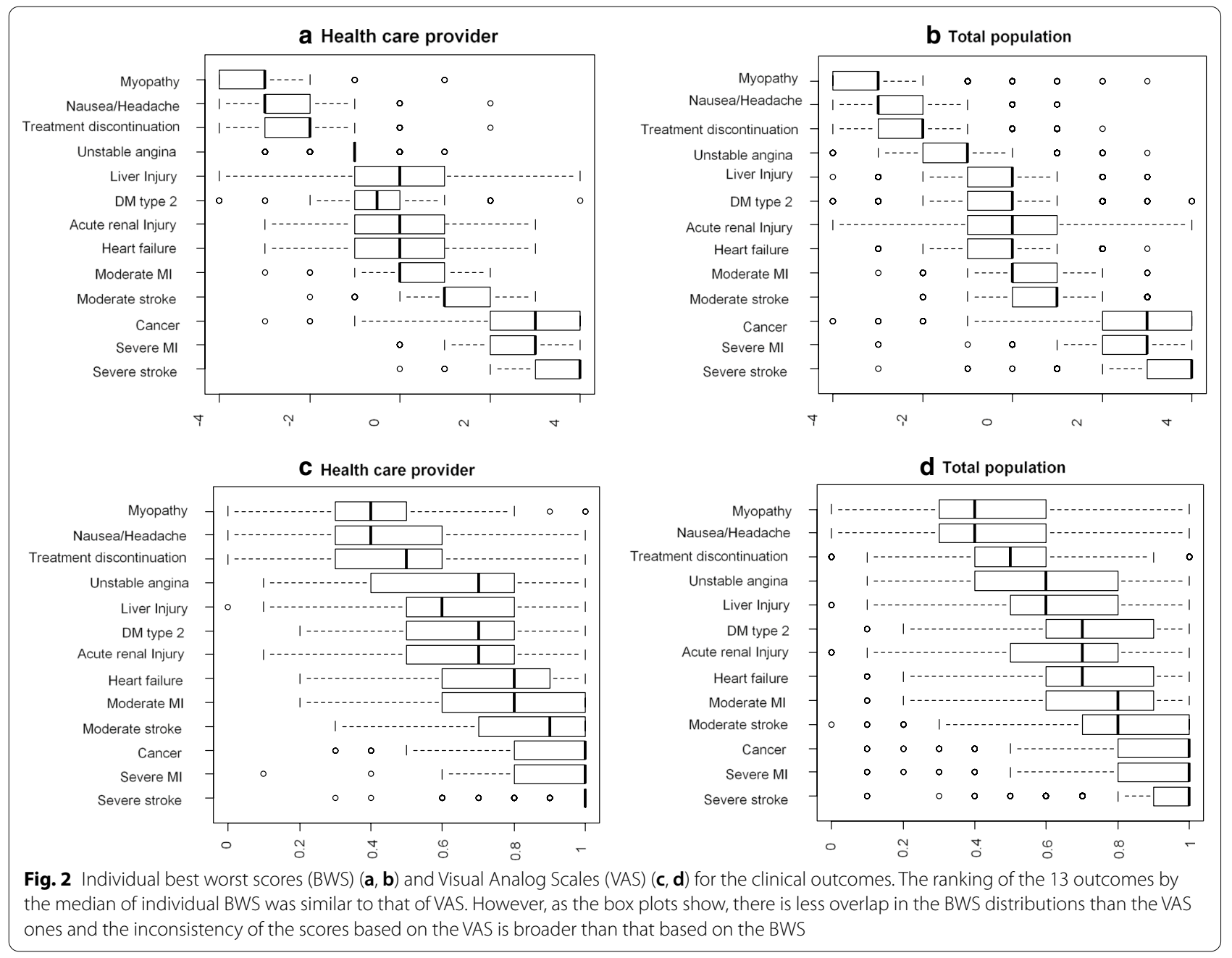

Table 3 Estimation results of Maximum-difference model. ${ }^{a}$

\begin{tabular}{lllll}
\hline & $\begin{array}{l}\text { Health care provider } \\
\mathbf{n = 2 6 , 8 3 2} \\
\text { Coefficient (95\% Cl) }\end{array}$ & $\begin{array}{l}\text { Total population } \\
\mathbf{n = 1 4 2 , 4 2 8} \\
\text { Coefficient } \mathbf{9 5 \%} \mathbf{~ C l})\end{array}$ & $\begin{array}{l}\text { Urban population } \\
\mathbf{n = 7 0 , 0 4 4} \\
\text { Coefficient (95\% Cl) }\end{array}$ & $\begin{array}{l}\text { Rural population } \mathbf{n = 7 2 , 3 8 4} \\
\text { Coefficient (95\% Cl) }\end{array}$ \\
\hline Severe stroke & $7.85(7.45-8.23)$ & $6.81(6.66-6.96)$ & $7.13(6.90-7.35)$ & $6.58(6.37-6.78)$ \\
Severe MI & $6.73(6.37-7.08)$ & $5.86(5.72-5.99)$ & $6.06(5.86-6.26)$ & $5.73(5.54-5.92)$ \\
Cancer & $6.62(6.26-6.97)$ & $5.76(5.63-5.90)$ & $6.28(6.07-6.48)$ & $5.34(5.16-5.52)$ \\
Moderate stroke & $5.41(5.08-5.75)$ & $4.52(4.39-4.65)$ & $4.91(4.72-5.10)$ & $4.20(4.03-4.37)$ \\
Moderate MI & $4.46(4.13-4.78)$ & $3.69(3.57-3.82)$ & $4.05(3.86-4.23)$ & $3.41(3.25-3.57)$ \\
Heart failure & $4.11(3.79-4.42)$ & $3.28(3.16-3.40)$ & $3.51(3.33-3.68)$ & $3.11(2.95-3.26)$ \\
Acute kidney failure & $4.16(3.84-4.48)$ & $3.27(3.15-3.39)$ & $3.58(3.40-3.76)$ & $3.02(2.86-3.18)$ \\
Type 2 diabetes & $3.60(3.28-3.92)$ & $3.18(3.06-3.30)$ & $3.32(3.13-3.50)$ & $3.08(2.92-3.25)$ \\
Liver injury & $4.04(3.72-4.36)$ & $2.93(2.81-3.05)$ & $3.26(3.08-3.44)$ & $2.67(2.51-2.82)$ \\
Unstable angina & $2.42(2.15-2.70)$ & $1.94(1.84-2.05)$ & $2.08(1.90-2.23)$ & $1.84(1.70-1.98)$ \\
Treatment discontinuation & $0.90(0.67-1.13)$ & $0.80(0.71-0.90)$ & $.90(0.76-1.04)$ & $.72(0.59-0.85)$ \\
Nausea/headache & $0.67(0.44-0.90)$ & $0.36(0.27-0.46)$ & $.30(0.16-0.44)$ & $.42(0.28-0.55)$ \\
Myopathy & Ref & Ref & Ref & Ref
\end{tabular}

a The coefficients are related to a conditional logit model. The exponential of the coefficients shows the odds ratio of choosing an item as the most worrisome outcome compared to choosing the Myopathy as the most worrisome one across all choice sets 
Table 4 Rating of the best-worst scaling exercise

\begin{tabular}{|c|c|c|c|c|c|}
\hline \multirow[t]{2}{*}{ Variable } & \multirow[t]{2}{*}{ Items } & \multicolumn{2}{|c|}{ Health care provider } & \multicolumn{2}{|c|}{ Total population } \\
\hline & & $\mathrm{N}=172$ & $\%$ & $\mathrm{~N}=913$ & $\%$ \\
\hline \multirow[t]{5}{*}{ Questions were easy to understand } & Strongly agree & 92 & 53.49 & 248 & 27.16 \\
\hline & Agree & 60 & 34.88 & 247 & 52.35 \\
\hline & Neither agree nor disagree & 9 & 5.23 & 147 & 16.10 \\
\hline & Disagree & 8 & 4.65 & 36 & 3.94 \\
\hline & Strongly disagree & 3 & 1.47 & 4 & 0.44 \\
\hline \multirow[t]{5}{*}{ Questions were easy to answer } & Strongly agree & 78 & 45.35 & 253 & 27.71 \\
\hline & Agree & 70 & 40.70 & 503 & 55.09 \\
\hline & Neither agree nor disagree & 12 & 6.98 & 123 & 13.47 \\
\hline & Disagree & 9 & 5.23 & 28 & 3.07 \\
\hline & Strongly disagree & 3 & 1.74 & 6 & 0.66 \\
\hline \multirow[t]{5}{*}{ Answers were consistent with my preferences } & Strongly agree & 100 & 58.14 & 384 & 42.06 \\
\hline & Agree & 64 & 37.21 & 486 & 53.23 \\
\hline & Neither agree nor disagree & 5 & 2.91 & 40 & 4.38 \\
\hline & Disagree & 3 & 1.74 & 3 & 0.33 \\
\hline & Strongly disagree & 0 & 0 & 0 & 0 \\
\hline \multirow{5}{*}{$\begin{array}{l}\text { Main reasons for influencing preference for health } \\
\text { problems }\end{array}$} & Availability of medical care & 41 & 23.84 & 285 & 31.22 \\
\hline & Severity of health problems & 45 & 26.16 & 223 & 24.42 \\
\hline & Curability & 34 & 19.77 & 134 & 14.68 \\
\hline & Long-term consequences & 33 & 19.19 & 117 & 12.81 \\
\hline & Cost-effectiveness of medical services & 19 & 11.05 & 154 & 16.87 \\
\hline
\end{tabular}

This table presents data on self-reported difficulties in understanding and compilation of the BWS questionnaire regarding different aspects

\section{Conclusion}

With the preference study results, we know how individuals consider the trade-offs between treatment benefits and harms. We found that our survey respondents consistently ranked severe stroke, severe MI, and cancers as the most worrisome outcomes while treatment discontinuation, nausea/headache, and myopathy as the least worrisome outcomes. The quantitative weights derived here for harms and benefits of statin therapy would be beneficial to determine the eligible people for statin therapy, mainly in a policy perspective. Our results showed that people from different socio-cultural contexts, rank the health outcomes the same way.

\section{Supplementary information}

Supplementary information accompanies this paper at https://doi. org/10.1186/s12911-020-01304-w.

Additional file 1.This contains supplementary tables and figures, including an example BWS questionnaire, balanced incomplete block design of outcomes, detailed results of counting analysis, Box plots of the individual BWS scores, results of linear regression assessing the influence of participants' characteristics on preference values, and the questionair

\section{Abbreviations}

BWS: Best-worst scaling;; LDL: Low-density lipoprotein; CVD: Cardiovascular disease; WHO: World health organization; VAS: Visual analog scale.

\section{Acknowledgements}

This study was conducted at the Research Institute for Endocrine Sciences, Shahid Beheshti University of Medical Sciences Tehran, Iran. Lown Scholar Program of the Harvard T.H. Chan School of Public Health helped in funding and designing the study. We thank the respectful staff of the Non-Communicable Diseases Office of the Ministry of Health and Medical Education in Iran for their help and advice on developing the survey. We gratefully thank Prof. MA Puhan for sharing their experience on the design and implementation of best-worst scaling.

\section{Authors' contributions}

All authors, HS, HRB, GD, AO, FH, LJ, EWS and DK contributed to the study conception and design. Material preparation and data collection were performed by DK, HS, HRB, and AO. HS and DK carried out data analysis, and GD, LJ and EWS provided critical comments on that. The first draft of the manuscript was written by $\mathrm{HS}$ and $\mathrm{DK}$ and $\mathrm{HRB}, \mathrm{GD}, \mathrm{AO}, \mathrm{FH}, \mathrm{LJ}$ and EWS commented on the manuscript. All authors read and approved the final manuscript.

\section{Funding}

This study is a part of a project funded by the National Institute of Medical Research. Development (NIMAD) (Grant No. 964114) and was supported by the Iran University of Medical. Sciences. The funding bodies had no role in the design of the study and collection, analysis, and interpretation of data and in writing the manuscript.

\section{Availability of data and materials}

The datasets used and/or analysed during the current study are available from the corresponding author on reasonable request. 


\section{Ethics approval and consent to participate}

Ethics approval for this study was obtained from the Ethics Committee of the Iran University of Medical Sciences (IR.IUMS.REC.1397.1073). Because the study did not involve any physical or laboratory examination, biological sampling and any respondents' identifiers, only oral consent was obtained from each participant after they were informed about the study's purpose and procedure, and as well as the confidentiality of data. The ethics committee approved this procedure.

\section{Consent for publication}

Not applicable.

\section{Competing interests}

The authors declare that there is no conflict of interest in this study.

\section{Author details}

${ }^{1}$ Department of Epidemiology, School of Public Health, Iran University of Medical Sciences, Tehran, Iran. ${ }^{2}$ Ageing Clinical and Experimental Research Team, Institute of Applied Health Sciences, School of Medicine, Medical Sciences and Nutrition University of Aberdeen, Aberdeen, UK. ${ }^{3}$ Endocrine Research Center, Institute of Endocrinology and Metabolism, Iran University of Medical Sciences, Tehran, Iran. ${ }^{4}$ Department of Global Health and Population and Department of Epidemiology, Harvard TH Chan School of Public Health, Boston, MA, USA. ${ }^{5}$ Osteoporosis Research Center, Endocrinology and Metabolism Clinical Sciences Institute, Tehran University of Medical Sciences, Tehran, Iran. ${ }^{6}$ Prevention of Metabolic Disorders Research Center, Research Institute for Endocrine Sciences, Shahid Beheshti University of Medical Sciences, Tehran, Iran. ${ }^{7}$ Department of Biostatistics, School of Public Health, Iran University of Medical Sciences, Tehran, Iran. ${ }^{8}$ Department of Biomedical Data Sciences, Leiden University Medical Center, Leiden, The Netherlands. ${ }^{9}$ Department of Public Health, Erasmus MC, Rotterdam, The Netherlands. ${ }^{10}$ Department of Biostatistics and Epidemiology, Research Institute for Endocrine Sciences, Shahid Beheshti University of Medical Sciences, Tehran, Iran.

Received: 10 July 2020 Accepted: 25 October 2020

Published online: 04 November 2020

\section{References}

1. Sarrafzadegan N, Mohammmadifard N: Cardiovascular Disease in Iran in the Last 40 Years: Prevalence, Mortality, Morbidity, Challenges and Strategies for Cardiovascular Prevention. Arch Iran Med. 2019, 22(4):204-210, https://www.ncbi.nlm.nih.gov/pubmed/31126179

2. Khalili D, Mosavi-Jarrahi A, Eskandari F, Mousavi-Jarrahi Y, Hadaegh F, Mohagheghi M, et al. Evaluation of cause of deaths'validity using outcome measures from a prospective, population based cohort study in Tehran Iran. PloS one. 2012. https://doi.org/10.1371/journal.pone.00314 27.

3. Catapano AL, Graham I, De Backer G, Wiklund O, Chapman MJ, Drexel H, et al. 2016 ESC/EAS guidelines for the management of dyslipidaemias. Eur Heart J. 2016;37(39):2999-3058. https://doi.org/10.1093/eurheartj/ ehw272.

4. Byrne P, Cullinan J, Smith A, Smith SM. Statins for the primary prevention of cardiovascular disease: an overview of systematic reviews. BMJ Open. 2019;9(4):e023085. https://doi.org/10.1136/bmjopen-2018-023085.

5. Stone NJ, Robinson JG, Lichtenstein AH, Bairey Merz CN, Blum CB, Eckel $\mathrm{RH}$ et al: 2013 ACC/AHA guideline on the treatment of blood cholesterol to reduce atherosclerotic cardiovascular risk in adults: a report of the American College of Cardiology/American Heart Association Task Force on Practice Guidelines. J Am Coll Cardiol. 2014, 63(25 Pt B):2889-2934. doi:https://doi.org/10.1016/j.jacc.2013.11.002

6. Cholesterol Treatment Trialists C, Baigent C, Blackwell L, Emberson J, Holland LE, Reith C et al: Efficacy and safety of more intensive lowering of LDL cholesterol: a meta-analysis of data from 170,000 participants in 26 randomised trials. Lancet. 2010, 376(9753):1670-1681. doi:https://doi. org/10.1016/S0140-6736(10)61350-5

7. Byrne P, Cullinan J, Murphy C, Smith SM. Cross-sectional analysis of the prevalence and predictors of statin utilisation in Ireland with a focus on primary prevention of cardiovascular disease. BMJ open. 2018;8(2):e018524. https://doi.org/10.1136/bmjopen-2017-018524.

8. Yebyo HG, Aschmann HE, Yu T, Puhan MA. Should statin guidelines consider patient preferences? Eliciting preferences of benefit and harm outcomes of statins for primary prevention of cardiovascular disease in the sub-Saharan African and European contexts. BMC Cardiovasc Disord. 2018;18(1):97. https://doi.org/10.1186/s12872-018-0838-9.

9. Naci H, Brugts J, Ades T. Comparative tolerability and harms of individual statins: a study-level network meta-analysis of 246955 participants from 135 randomized, controlled trials. Circ Cardiovasc Qual Outcomes. 2013;6(4):390-9. https://doi.org/10.1161/CIRCOUTCOMES.111.000071.

10. Chou R, Dana T, Blazina I, Daeges M, Jeanne TL. Statins for prevention of cardiovascular disease in adults: evidence report and systematic review for the US preventive services task force. JAMA. 2016;316(19):2008-24. https://doi.org/10.1001/jama.2015.15629.

11. Elwyn G, Frosch D, Rollnick S. Dual equipoise shared decision making: definitions for decision and behaviour support interventions. Implement Sci. 2009;4(1):75. https://doi.org/10.1186/1748-5908-4-75.

12. Carling $\mathrm{CL}$, Kristoffersen DT, Montori VM, Herrin J, Schünemann $\mathrm{HJ}$, Treweek S, et al. The effect of alternative summary statistics for communicating risk reduction on decisions about taking statins: a randomized trial. PLOS Medicine. 2009;6(8):e1000134. https://doi.org/10.1371/journ al.pmed.1000134.

13. Lloyd-Jones DM, Braun LT, Ndumele CE, Smith SC Jr, Sperling LS, Virani SS, et al. Use of risk assessment tools to guide decision-making in the primary prevention of atherosclerotic cardiovascular disease: a special report from the American heart association and american college of cardiology. Circulation. 2019;139(25):e1162-77. https://doi.org/10.1161/ CIR.0000000000000638

14. Martin SS, Sperling LS, Blaha MJ, Wilson PWF, Gluckman TJ, Blumenthal RS, et al. Clinician-patient risk discussion for atherosclerotic cardiovascular disease prevention: importance to implementation of the 2013 ACC/AHA Guidelines. J Am Coll Cardiol. 2015;65(13):1361-8. https://doi. org/10.1016/j.jacc.2015.01.043.

15. Cheung KL, Wijnen BF, Hollin IL, Janssen EM, Bridges JF, Evers SM, et al. Using best-worst scaling to investigate preferences in health care. Pharmacoeconomics. 2016;34(12):1195-209. https://doi.org/10.1007/s4027 3-016-0429-5.

16. Finn A, Louviere JJ. Determining the appropriate response to evidence of public concern: the case of food safety. J Public Policy Mark. 1992;11(2):12-25. https://doi.org/10.1177/074391569201100202.

17. Louviere JJ, Flynn TN. Using best-worst scaling choice experiments to measure public perceptions and preferences for healthcare reform in Australia. Patient Patient-Centered Outcomes Res. 2010;3(4):275-83. https ://doi.org/10.2165/11539660-000000000-00000.

18. Flynn TN. Valuing citizen and patient preferences in health: recent developments in three types of best-worst scaling. Expert Rev Pharmacoecon Outcomes Res. 2010;10(3):259-67. https://doi.org/10.1586/erp.10.29.

19. Mühlbacher AC, Kaczynski A, Zweifel P, Johnson FR. Experimental measurement of preferences in health and healthcare using best-worst scaling: an overview. Health Econ Rev. 2016;6(1):2. https://doi.org/10.1186/ s13561-015-0079-x.

20. Word Health Organization, 2017. Islamic Republic of Iran on a fast-track to beating noncommunicable diseases. WHO. [https://www.who.int/ news-room/feature-stories/detail/islamic-republic-of-iran-on-a-fast-track -to-beating-noncommunicable-diseases.]

21. Aizaki H: support.BWS: Tools for Case 1 Best-Worst Scaling. 2020, https:// cran.r-project.org/web/packages/support.BWS/index.html

22. Goff DC, Lloyd-Jones DM, Bennett G, Coady S, D'agostino RB, Gibbons R et al: 2013 ACC/AHA guideline on the assessment of cardiovascular risk: a report of the American College of Cardiology/American Heart Association Task Force on Practice Guidelines. J Am Coll Cardiol. 2014, 63(25 Part B):2935-2959. doi:https://doi.org/10.1016/j.jacc.2013.11.005

23. Shuhaili MFRMA, Samsudin IN, Johnson Stanslas SH, Thambiah SC: Effects of different types of statins on lipid profile: a perspective on Asians. Int J Endocrinol Metab. 2017, 15(2). doi:https://doi.org/10.5812/ijem.43319

24. Board JBS: Joint British Societies' consensus recommendations for the prevention of cardiovascular disease (JBS3). Heart. 2014, 100 Suppl 2(Suppl 2):ii1-ii67. doi:https://doi.org/10.1136/heartjnl-2014-305693

25. Puhan MA, Singh S, Weiss CO, Varadhan R, Boyd CM. A framework for organizing and selecting quantitative approaches for benefit-harm 
assessment. BMC Med Res Methodol. 2012;12(1):173. https://doi. org/10.1186/1471-2288-12-173.

26. MacLean S, Mulla S, Akl EA, Jankowski M, Vandvik PO, Ebrahim S, et al. Patient values and preferences in decision making for antithrombotic therapy: a systematic review: antithrombotic therapy and prevention of thrombosis, 9th ed: American College of Chest Physicians EvidenceBased Clinical Practice Guidelines. Chest. 2012;141(2 Suppl):e1S-e23S. https://doi.org/10.1378/chest.11-2290.

27. Blinman P, King M, Norman R, Viney R, Stockler MR. Preferences for cancer treatments: an overview of methods and applications in oncology. Ann Oncol. 2012;23(5):1104-10. https://doi.org/10.1093/annonc/mdr559.

28. Ho M, Saha A, McCleary KK, Levitan B, Christopher S, Zandlo K, et al. A Framework for incorporating patient preferences regarding benefits and risks into regulatory assessment of medical technologies. Value Health. 2016;19(6):746-50. https://doi.org/10.1016/j.jval.2016.02.019.

29. Muhlbacher AC, Juhnke C, Beyer AR, Garner S. Patient-focused benefit-risk analysis to inform regulatory decisions: the european union perspective. Value Health. 2016;19(6):734-40. https://doi.org/10.1016/j. jval.2016.04.006

30. Yebyo HG, Aschmann HE, Puhan MA. Finding the balance between benefits and harms when using statins for primary prevention of cardiovascular disease: a modeling study. Ann Intern Med. 2019;170(1):1-10. https ://doi.org/10.7326/M18-1279.
31. Ali S, Ronaldson S. Ordinal preference elicitation methods in health economics and health services research: using discrete choice experiments and ranking methods. Br Med Bull. 2012;103(1):21-44. https://doi. org/10.1093/bmb/lds020.

32. Potoglou D, Burge P, Flynn T, Netten A, Malley J, Forder J et al: Best-worst scaling vs. discrete choice experiments: an empirical comparison using social care data. Social Sci Med. 2011, 72(10):1717-1727. doi:https://doi. org/10.1016/j.socscimed.2011.03.027

33. Flynn TN, Louviere JJ, Peters TJ, Coast J. Best-worst scaling: what it can do for health care research and how to do it. J Health Econ. 2007;26(1):17189. https://doi.org/10.1016/j.jhealeco.2006.04.002.

34. Meara A, Crossnohere NL, Bridges JF. Methods for measuring patient preferences: an update and future directions. Curr Opin Rheumatol. 2019;31(2):125-31. https://doi.org/10.1097/BOR.0000000000000587.

\section{Publisher's Note}

Springer Nature remains neutral with regard to jurisdictional claims in published maps and institutional affiliations.
Ready to submit your research? Choose BMC and benefit from:

- fast, convenient online submission

- thorough peer review by experienced researchers in your field

- rapid publication on acceptance

- support for research data, including large and complex data types

- gold Open Access which fosters wider collaboration and increased citations

- maximum visibility for your research: over $100 \mathrm{M}$ website views per year

At BMC, research is always in progress.

Learn more biomedcentral.com/submissions 